\title{
Nisis $T=1.70779$
}

\section{PROTONS AS THE PRIME CONTRIBUTORS TO THE STORM TIME RING CURRENT}

(NASA-TH-X-70779) PROTONS AS THE PRIME CONTRIBUTORS TO THE STORM TIME RING CORRENT (NASA) $19 \mathrm{p}$ HC $\$ 3 .-25$ CSCL $20 \mathrm{H}$

\author{
N75- 1.1722

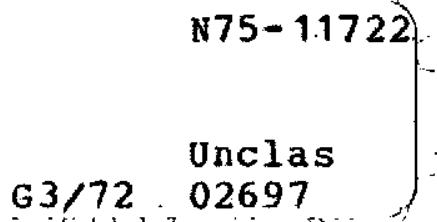

F. W. BERKO

L. J. CAHILL, JR.

T. Á. FRITZ

OCTOBER 1974 
"This paper presents the views of the author(s), and does not necessarily reflect the views of the Goddard Space

Flight Center, or NASA."

For information concerning availability of this document contact:

Technical Information Division, Code 250

Goddard Space-Flight Center

Greenbelt, Maryland 20771

(Telephone 301-982-4488) - 
PROTONS AS THE PRTIE CONTRIBUTORS

TO STORV TTIE RTHE CURRENT

F. W. Berko

Goddard Space Flight Center, Greenbelt, Ma. 20771

L. J. Cahill, Jr.

University of Minnesota, Minneapolis, Minn. 55455

T. A. Fritz

NOAA/SEL, Bounder, Colorado 80302 
Following a large sudden conmencement on 17 June 1972, a large magnetic storm evolved, with a well-developed main phase and recovery phase. Explorer $45\left(S^{3}-A\right)$, with its apogee near 16 hours local time in June, measured the equatorial particle populations and magnetic field throughout this period. Using data obtained during the symmetric recovery phase, it is shown that, through a series of self-consistent calculations, the measured protons, with energies from $1 \mathrm{keV}$ to $872 \mathrm{keV}$, can account for the observed ring current magnetic effects within experi mental uncertainties. This enables us to set an upper limit to the heavy ion contribution to the storm time ring current of a few percent of the proton contribution. 
Introduction

Explorer 45 was launched into an elliptical, equatorial orbit on 15 November, 1971. It carried a variety of instruments which made extensive measurements of the ring current and other phenomena in the inner magnetosphere (Longanecker and Hoffman, 1973). Initial data from Explorer 45 have been presented showing magnetic fileld measurements (Cahill, 1973), ring current particle distributions (Smith and Hoffman, 1973), and proton energy spectra and pitch angle distributions (Konradi et al., 1973). Hoffman (1973) has presented simultaneous particle and magnetic field data obtained by $\mathrm{S}^{3}-\mathrm{A}$ during the December 1971 magnetic storm period.

Data from the Explorer 45 satellite will be used in this paper to calculate the deformation of the geomagnetic fleld due to ring current protons, using the self-consistent technique of Hoffman and Bracken (1967). Previously, using the Hoffman and Bracken calculation method, Hof'man and Cahill (1968) used Explorer 26 magnetometer data to derive a ring current particle distribution which gave good agreement with the magnetic observations. Prior to Explorer 45 ring current measurements, only one case of direct observations of the storm-time ring current particle system was reported (Frank, 1967). Smith and Hoffman (1974) have recently discussed several cases of storm-time ring current particle measurements made by the $S^{3}-A$ satellite in the dusk hours. Data to be used in this study were collected when the spacecraft apogee was at about 16 hours MLT.

\section{Magnetic Conditions and Reguirements}

The first figure presents the equatorial Dst values for June 17 - 
20, 1972, with day and time indicated on the bottom and the $S^{3}-A$ satellite orbit numbers during this time indicated along the top. As is evident from the figure, this magnetic storm was characterized by a very large main phase, with Dst reaching a minimum value of -1908; and a long recovery phase extending at least one day past the end of the figure. To perform the ring current calculations to be described, we sought a time period when the following conditions prevailed:

i) The proton energy density measured on the inbound and outbound portions of the $(7+$ hour $)$ orbit were symmetric;

ii) The satellite orbit remained very close (i.e. within $\pm 1^{\circ}$ ) to. the magnetic equator;

iii) The satellite apogee was on the dayside;

Iv) The $\triangle B$ profile obtained from the onboard magnetometer was essentially symmetric on the outbound and inbound portions of the orbit, implying the existence of a symmetric ring current.

Orbit 676 , on June 19, satisfied all these requirements, and data from this orbit will be used in this study. These data were obtained during the symmetric recovery phase, at a time of little substorm activity. Measurements made during substorms showed more complicated effects (Williams et al., 1974) and are unsuitable for the calculations to be performed here.

\section{Particle and Magnetic Observations}

Figure 2 presents the differential energy spectra at three I 
values for $1 \mathrm{kev}$ to $872 \mathrm{kev}$ protons during orbit 676. The spectra are averaged over both outbound and inbound data obtained at $L$ values of $2.5,3.5$ and 4 . Note first that the maximum energy density was at $L=3.5$. Energy spectra for $L=3.5$ and 4 are quite similar. They both decrease fairly uniformly from 1 kev to about $120 \mathrm{kev}$, then decrease very rapidly at energies above $120 \mathrm{kev}$. At $I=2.5$ the spectrum is considerably less intense and decreases more rapidly up to about $220 \mathrm{kev}$ than the other two spectra, but then remains essentially flat up to the maximum energy of $872 \mathrm{kev}$.

Pitch angle distributions of the measured protons are presented In Figure 3 at $L$ values of $2.5,3.5$ and 4 for data obtained both on the outbound (circles) and inbound (triangles) portions of orbit. 676. The distributions at each $L$ value were then fit to a function of the form $\sin ^{n} \alpha$. At $L=2.5, n$ had the value 2.3, while at $L=4, n$ was 1.3. It was determined that $n$ varled linearly from $L=2.5$ to $L=4$, and then $n$ remained approximately constant at 1.3 for $L$ values greater than 4, (at least out to $S^{3}-A$ apogee at $L=5.2$ and 16 hours local time).

Measured proton energy densities over the range 1 kev to 872 kev from inbound and outbound portions of orbit 676 were integrated over energy and pitch angle and then averaged. The resulting values are plotted as a function of $L$ in Figure 4. A smooth, continuously differentiable function composed of a combination of exponentials and fifth-order polynomials was then fit to the data, with special attention given to fitting the data both in value and slope near the 
maximum energy density at $L=3.5$. This function is shown as the solid curve in figure 4, and is seen to fit measurements very well at $\mathrm{L}$ values from 3 to 4.8 .

Inbound and outbound magnetometer values were averaged to obtain the (dashed) curve shown in Figure 5 as the measured $\Delta \mathrm{B}$ (see e.g. Cahill, 1973; Hoffman, 1973). There was a slight asymmetry in measured $\Delta B$ at the minimum value near $L=3.4$. On the outbound pass the minimum value was approximately -115 gammas; inbound it was approximately -130 garmas. $\Delta B$ is defined as

$$
\Delta B=\left|\vec{B}_{\text {measured }}\right|-\left|\vec{B}_{\text {model }}\right|
$$

Calculational Approach

The smooth energy density profile of figure 4 and the pitch angle distributions presented above were then used to calculate the magnetic effects of the proton ring current in a self-consistent manner (see Hoffman and Bracken, 1967):

i) The proton energy density and pitch angle distributions were placed in a model magnetic fleld composed of a main (spherical harmonic expansion) field plus boundary and neutral sheet effects. The current resulting from these particles in this field model produced a $1^{\text {st }}$ order magnetic field, from which was obtained a first order $\Delta B$.

ii) This $1^{\text {st }}$ order ring current field was added vectorially to the (original) model field. A $2^{\text {nd }}$ order ring current was then calculated in this $1^{\text {st }}$ order field from the measured proton energy density, and then a $2^{\text {nd }}$ order ring current 
field was computed.

iii) This procedure was repeated until self-consistency was attained, that is when $\Delta B\left(n^{\text {th }}\right.$ order) was essentially equal to $\Delta B\left(n+1^{\text {st }}\right.$ order $)$. In this case, the $3^{\text {rd }}$ and $4^{\text {th }}$ order $\Delta B$ differed by less than $1 \%$.

Both the $I^{\text {St }}$ and $3^{\text {rd }}$ order $\Delta B$ profiles are shown in Figure 5. Differences between the measured and $3^{\text {rd }}$ order calculated $\Delta B$ are less than $10 \%$ from $L=2$ to $L=5$, and can be accounted for largely by uncertainties in the measurements and inaccuracies in the fit to the measured energy density profile (figure 4).

\section{DISCUSSION}

We have shown that, within experimental uncertainties and inaccuracies to the fitted data, we can account for the observed $\Delta \mathrm{B}$ with the measured count rates. However, one must consider the possibility that at least some of the counts recorded in our particle detectors might have been due to heavy 1ons. Shelley et al., (1972) have reported observations of heavy ions from low altitude spacecraft, and have suggested that these heavy ions could make "... a substantial contribution to the storm-time magnetic-field depression (Dst)...", noting that, for the same proton and oxygen fluxes, the $\mathrm{O}^{+}$energy density is 4 times the proton energy density. More recently, Johnson et al. (1974) have reported fluxes of $\mathrm{He}^{+}$ions, also observed at low altitudes, that are not insignificant, and which they belleve to be of ionospheric origin. 
Thus, it is of interest to examine what the $S^{3}-A$ megnetic and particle data can say with regard to the heavy ion contribution to the ring current during orbit 676 . If we assume that $5 \%$ of the counts in the particle detectors were due to heavy ions $\left(\mathrm{O}^{+}\right)$, and further that these ions had the same pitch angle distribution as the protons, then the energy density curve in Figure 4 would be increased by 15\%. It follows then that using this higher value for the energy density in the same type of self-consistent calculation, we should arrive at values of $\Delta B$ which are about 12\% greater than those calculated using the original energy density profile. At this point, we must examine the uncertainties that exist in the "measured $\Delta B^{\prime}$. Uncertainties in the magnetometer measurements, due to averaging over time and inbound and outbound data, are on the order of 58 . In the determination of the measured $\triangle B$ curve, a model field was used, from which the measurements were subtracted. The absolute validity of this model field will not be discussed here, but its relative accuracy is important. The model used is based on measurements made under a variety of magnetic conditions. It has several adjustable parameters (i.e. subsolar distance of the magnetopause, epoch) which have effects on the magnitude of the field it returns at any given $L$, latitude and longitude. Variations in these parameters can change the model field values by as much as $10 \mathrm{r}$ in the ring current region. Thus, the combined uncertainty in the measured $\Delta B$ profile is of the order of $15 \gamma$. Consequently, a change of about $12 \%$ in the calculated, self-consistent $\Delta B$ is within the uncertainty which we can assign to the measured $\triangle B$. 
Although the field model used for the ring current calculations includes effects of distributed currents in the magnetosphere (Olson, 1974), the model used for determining the measured $\Delta \mathrm{B}$ does not. However, since we have chosen primarlly dayside data, effects of neutral sheet and tail current fields are minimal, and the greatest effects of magnetopause currents are at $L=5$, decreasing at smaller $L$ values.

We can therefore place an upper limit to the ion flux in the ring current region at a few percent of the proton flux at the time of orbit 676. A confirmation of the low heavy ion contribution is also indicated by the low $\mathrm{O}^{+}$precipitation observed at this time by the 1971-089A mass spectrometer (R. D. Sharp, private communication, 1974). It should be noted that earlier in this storm, during the asymmetric phase, definite $\mathrm{O}^{+}$and $\mathrm{He}^{+}$precipitation was observed in the 1971 089A data.

\section{CONCLUSIONS}

We have shown that when the satellite orbit was equatorial during a magnetic storm recovery phase and the magnetometer indicated the existence of a symmetric ring current, the measured proton energy density could be used to calculate a $\Delta \mathrm{B}$ profile which is in good agreement with the measured profile. As a corollary, we can therefore place an upper limit to the ion flux in the ring current region at a few percent of the proton flux,

\section{ACKNOWLEDGENENTS}

We are pleased to acknowledge the helpful discussions and 
$-8-$

beneficial criticisms from R. A. Hoffman and P. H. Smith. R. D. Sharp made many help $\mathbb{1}$ suggestions in a critical reading of the manuscript. 


\section{REFERENCES}

Cahili, I. J., Jr., Magnetic Storm Inflation in the Evening Sector, JGR, 78, 4724-4730, 1973.

Frank, L. A., On the Extra-terrestrial Ring Current During Geomagnetic Storms, JGR, 72, 3753-3767, 1967.

Hoffman, R. A., Particle and Field Observations from Explorer 45

During the December 1971 Magnetic Storm Period, JGR, 78, 47714777, 1973.

Hoffman, R. A., and Bracken, P. A., Higher-order Ring Currents and Particle Energy Storage in the Magnetosphere, JGR, 72, 6039$6049,1967$.

Hoffman, R. A., and Cahill, L. J.,Jr., Ring Current Particle Distribution Derived from Magnetic Fleld Measurements, JGR, 73, 6711-6722, 1968. Johnson, R. G., Sharp, R. D., and Shelley, E. G., The Discovery of Energetic $\mathrm{He}^{+}$Ions in the Magnetosphere, J. Geophys. Res., 79, $3135-3139,1974$.

Konradi, A., Williams, D. J., and Fritz, T. A., Energy Spectra and Pitch Angle Distributions of Storm-time and Substorm Injected Protons, JGR, 78, 4739-4744, 1973.

Longanecker, G. W., Hoffman, R. A., $S^{3}-A$ Spacecraft and Experiment Description, JGR, 78, 4711-4717, 1973. Olson, W. P., A Model of the Distributed Magnetospheric Currents, J. Geophys. Res., 79, 3731-3738, 1974. Shelley, E. G., Johnson, R. G. and Sharp, R. D., Satellite Observations of Energetic Heavy Ions During a Geomagnetic Storm, JGR, $\underline{77}$, 6104-6110, 1972. 
Smith, P. H. and Hoffman, R. A., Ring Current Particle Distributions During the Magnetic Storms of December 16-18, 1971, J. Geophys. Res., 78, 4731-4737, 1973.

Snith, P. H. and Hoffman, R. A., Direct Observations in the Dusk Hours of the Characteristics of the Storm Time Ring current Particles During the Beginning of Magnetic Storms, JGR, 79, $966-971,1974$.

Williams, D. J., Barfield, J. N. and Fritz, T. A., Initial Explorer 45 Substorm Observations and Electric Field Considerations, JGR, 79, 554-564, 1974 . 
Figure 1: Equatorial values of $\mathrm{D}_{\mathrm{ST}}$ for the period 17 to 20 June, 1972. $\mathrm{S}^{3}-\mathrm{A}$ orbit numbers are shown at the top.

Figure 2: Differential energy spectra averaged over inbound and outbound data at three $L$ values.

Figure 3: Pitch angle distributions at three $L$ values for both outbound and inbound data during orbit 676 .

Figure 4: Proton energy densities integrated over energy and pitch angle and averaged over inbound and outbound portions of orbit 676; a smooth, differentiable function fit to these data is shown as the solid curve.

Figure 5: Measured $\Delta B$ (dashed curve) and $1^{\text {st }}$ and $3^{\text {rd }}$ order calculated $\Delta B$ profiles. 


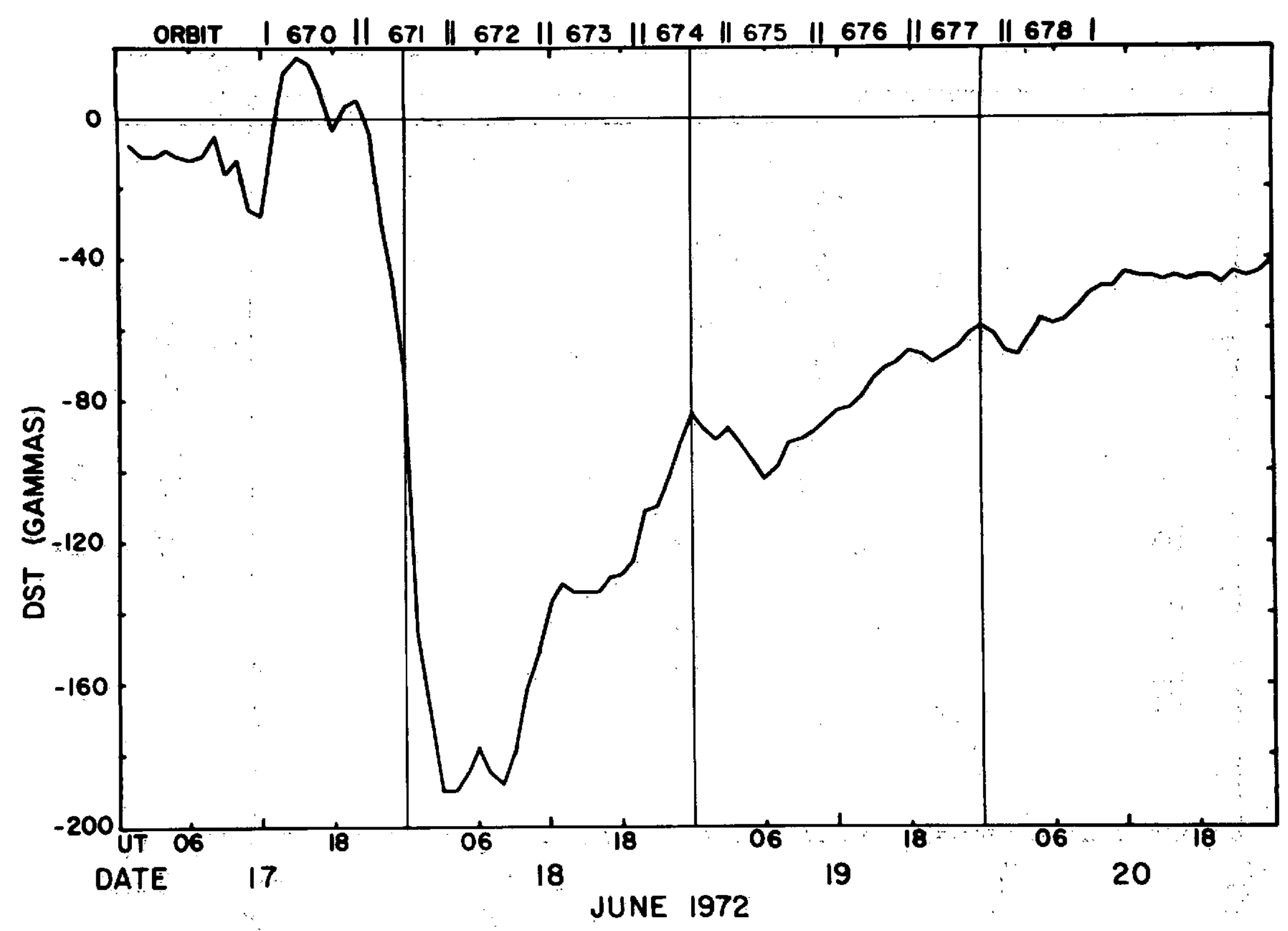

Figure 1 


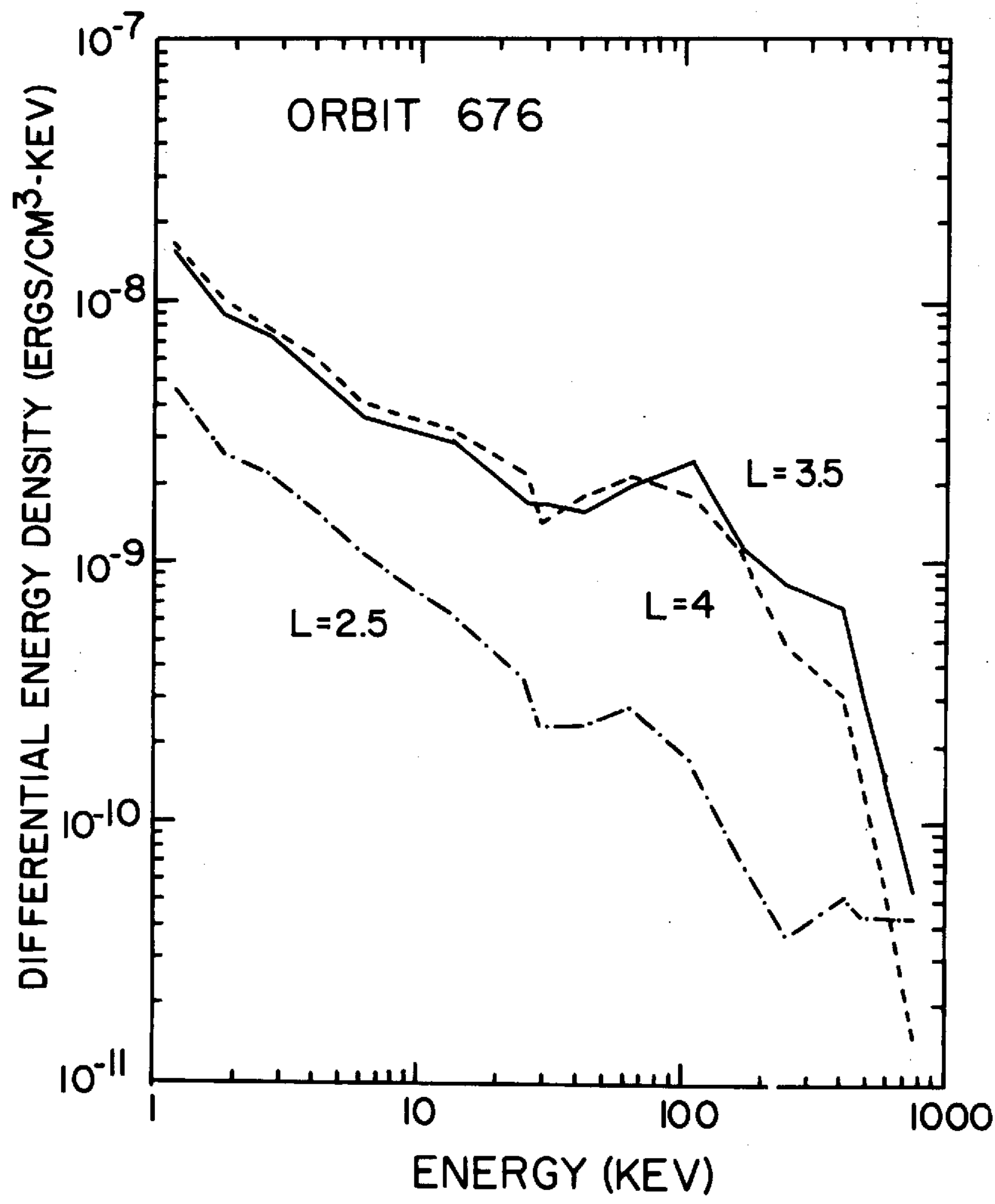

Figure 2 


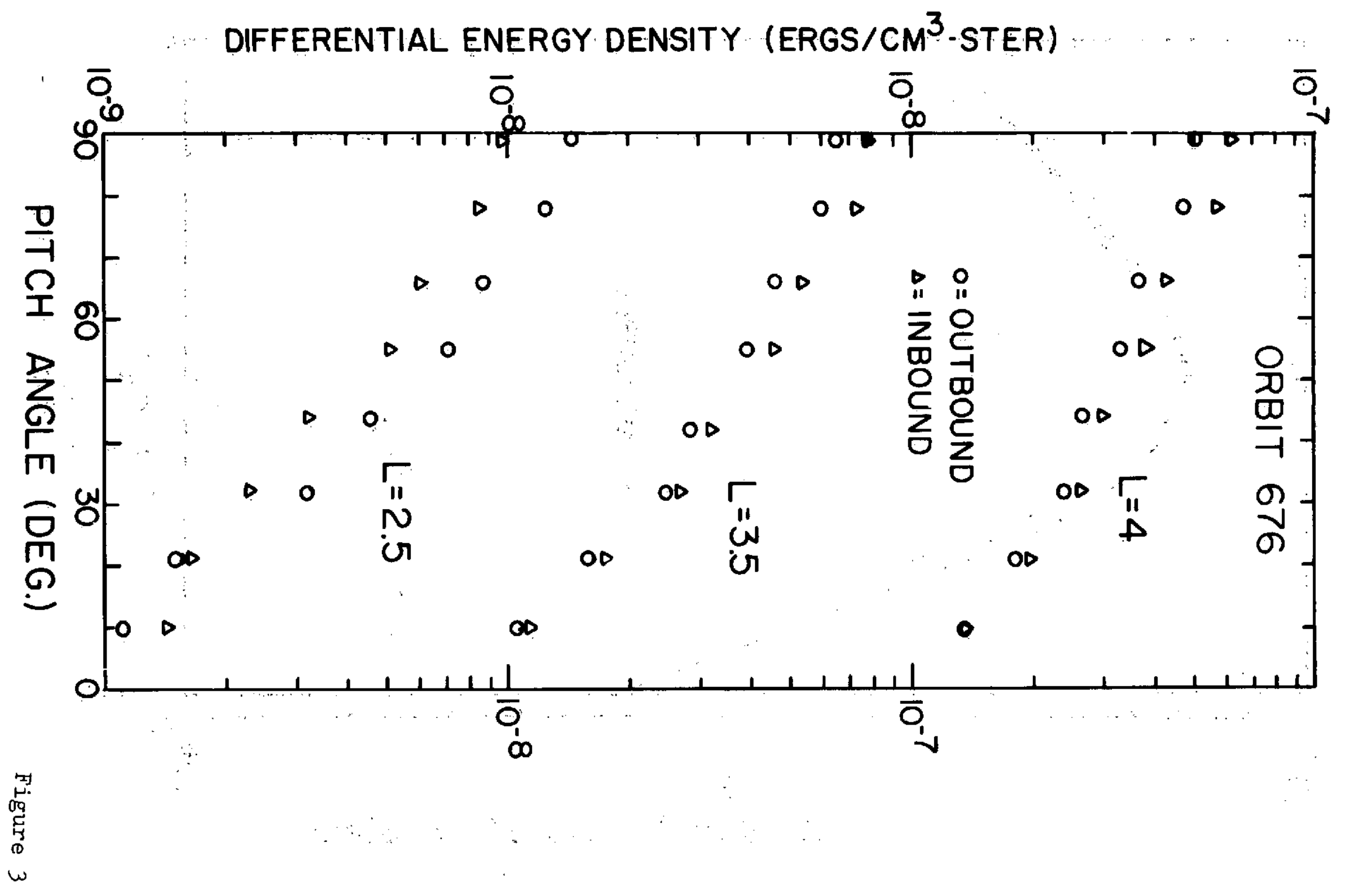




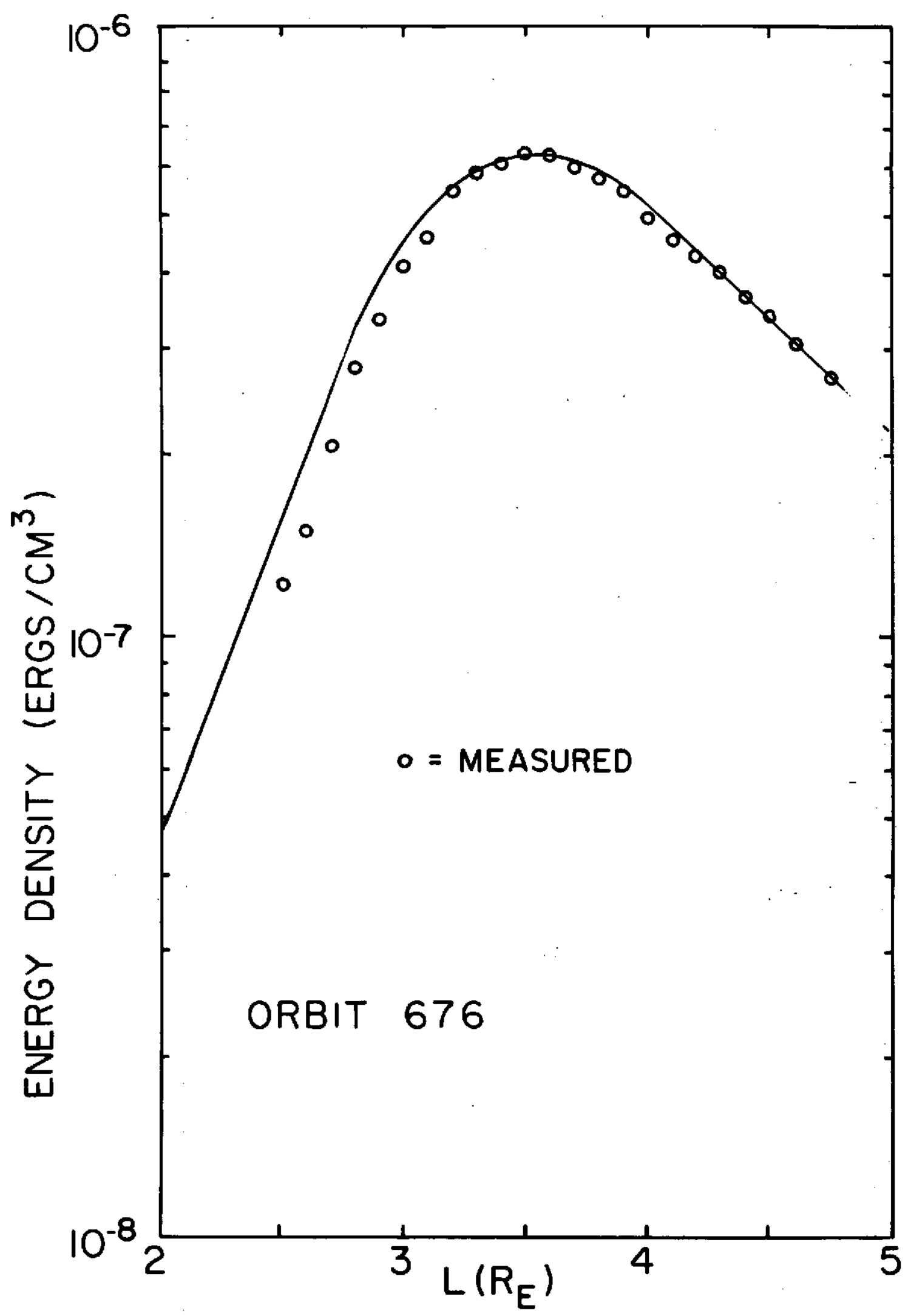

Figure 4 


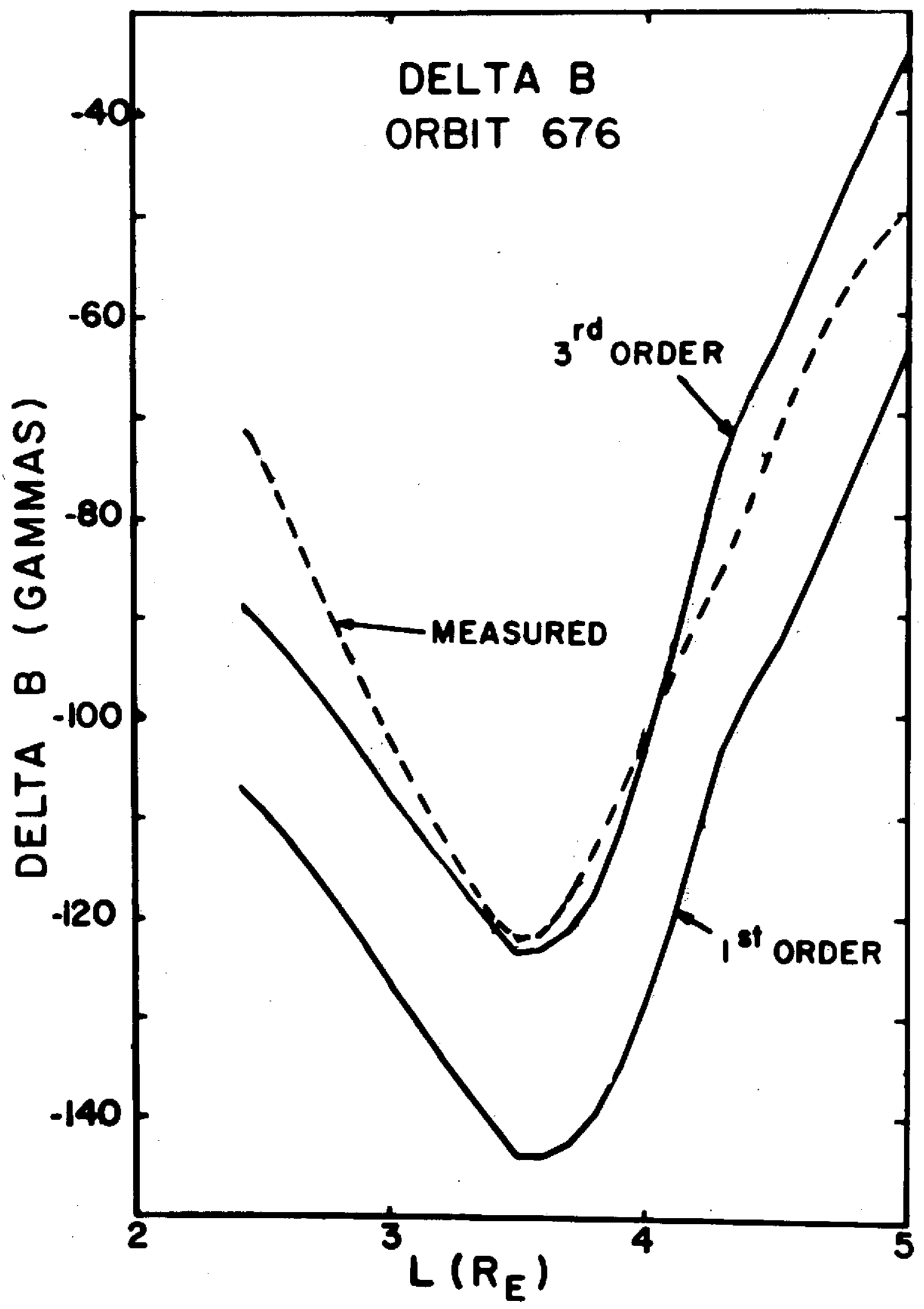

Figure 5 